\title{
Lumbar Lateral Interbody Fusion (LLIF): Comparative Effectiveness and Safety versus PLIF/TLIF and Predictive Factors Affecting LLIF Outcome
}

\author{
Giuseppe M. V. Barbagallo ${ }^{1} \quad$ Vincenzo Albanese ${ }^{1}$ Annie L. Raich ${ }^{2}$ Joseph R. Dettori ${ }^{2}$ Ned Sherry $^{2}$ \\ Massimo Balsano 3
}

${ }^{1}$ Division of Neurosurgery, Department of Neurosciences, Policlinico University Hospital, Catania, Italy

${ }^{2}$ Spectrum Research, Inc., Tacoma, Washington, United States

${ }^{3}$ Regional Spinal Department, Alto Vicentino, OC Santorso,

Vicenza, Italy

Evid Based Spine Care J 2014;5:28-37.

\begin{abstract}
Address for correspondence Giuseppe M. V. Barbagallo, MD, Division of Neurosurgery, Department of Neurosciences, Policlinico University Hospital, Via S. Sofia 78, Catania, Italy (e-mail: giuseppebarbagal@hotmail.com).
\end{abstract}

\begin{abstract}
Keywords

- XLIF ${ }^{\circledR}$

- DLIF

- LLIF

- PLIF

- TLIF

- minimally invasive

- extreme lateral interbody fusion

- direct lateral interbody fusion

Study Design Systematic review.

Study Rationale The surgical treatment of adult degenerative lumbar conditions remains controversial. Conventional techniques include posterior lumbar interbody fusion (PLIF) or transforaminal lumbar interbody fusion (TLIF). A new direct approach known as lumbar lateral interbody fusion (LLIF), or extreme lateral interbody fusion $\left(\mathrm{XLIF}^{\circledR}\right)$ or direct lateral interbody fusion (DLIF), has been introduced.

Objectives The objective of this article is to determine the comparative effectiveness and safety of LLIF, at one or more levels with or without instrumentation, versus PLIF or TLIF surgery in adults with lumbar degenerative conditions, and to determine which preoperative factors affect patient outcomes following LLIF surgery.

Materials and Methods A systematic review of the literature was performed using PubMed and bibliographies of key articles. Articles were reviewed by two independent reviewers based on predetermined inclusion and exclusion criteria. Each article was evaluated using a predefined quality rating scheme.

Results The search yielded 258 citations and the following met our inclusion criteria: three retrospective cohort studies (all using historical cohorts) (class of evidence [CoE] III) examining the comparative effectiveness and safety of LLIF/XLIF ${ }^{\circledR} /$ DLIF versus PLIF or TLIF surgery, and one prospective cohort study (CoE II) and two retrospective cohort studies (CoE III) assessing factors affecting patient outcome following LLIF. Patients in the LLIF group experienced less estimated blood loss and a lower mortality risk compared with the PLIF group. The number of levels treated and the preoperative diagnosis were significant predictors of perioperative or early complications in two studies.

Conclusion There is insufficient evidence of the comparative effectiveness of LLIF versus PLIF/TLIF surgery. There is low-quality evidence suggesting that LLIF surgery results in fewer complications or reoperations than PLIF/TLIF surgery. And there is insufficient evidence that any preoperative factors exist that predict patient outcome after LLIF surgery.
\end{abstract}

received

September 3, 2013

accepted after revision

December 17, 2013 (c) 2014 Georg Thieme Verlag KG

Stuttgart · New York
DOI http://dx.doi.org/ 10.1055/s-0034-1368670. ISSN 1663-7976. 


\section{Study Rationale and Context}

The surgical treatment of adults degenerative lumbar conditions remains very controversial. Lumbar interbody arthrodesis, with or without instrumentation, provides better fusion rate but not better clinical results.

To reduce surgical morbidity and achieve satisfactory, long-standing results, a new direct approach to the lumbar spine, known as lumbar lateral interbody fusion (LLIF), direct lateral interbody fusion (DLIF), or extreme lateral interbody fusion $\left(\mathrm{XLIF}^{\circledR}\right.$; Nuvasive, San Diego, CA, United States), has been introduced.

A comparative analysis of this new approach versus conventional posterior lateral interbody fusion (PLIF) or transforaminal lumbar interbody fusion (TLIF) techniques is the aim of this study.

\section{Objectives}

The objective of this article is to determine the following:

- The comparative effectiveness and safety of LLIF, XLIF, or DLIF surgery at one or more levels with or without instrumentation versus PLIF or TLIF surgery, in adults with lumbar degenerative conditions including degenerative scoliosis

- What preoperative factors, if any, affect patient outcomes following LLIF, XLIF, or DLIF surgery.

\section{Materials and Methods}

Study design: This study is a systematic review.

Search: The databases included PubMed, Cochrane, and National Guideline Clearinghouse Databases, as well as bibliographies of key articles.

Dates searched: The dates were searched till November 2013. Inclusion criteria: The inclusion criteria of the study were as follows: (1) patients 18 years or older, (2) lumbar degenerative disc disease (DDD) (with or without canal stenosis and with or without degenerative spondylolisthesis) or lumbar degenerative scoliosis, (3) studies with at least 10 patients per treatment group (comparative effectiveness) or studies with at least 20 patients total (predictive factors), and (4) comparison of LLIF/XLIF/DLIF with PLIF/TLIF surgery (comparative effectiveness).

Exclusion criteria: The exclusion criteria of the study were as follows: (1) patients younger than 18 years, (2) those involving traumatic onset, fracture, thoracic disc disease, infection, or neoplasms; (3) case reports, comparative studies with fewer than 10 patients per treatment group; and (4) cadaveric studies, nonhuman in vivo, in vitro, and biomechanical studies.

Outcomes: The outcomes of the study include the following: (1) perioperative complications, (2) reoperation risk, (3) complications or adverse events, (4) postoperative pain, (5) neurological improvement, and (6) sagittal and coronal balance.
Analysis: Descriptive statistics. Pooling of data was not done due to concerns regarding heterogeneity of treatments and populations as well as study quality.

Overall strength of evidence: Risk of bias for individual studies was based on using criteria set by The Journal of Bone and Joint Surgery ${ }^{1}$ modified to delineate criteria associated with methodological quality and risk of bias based on recommendation from the Agency for Healthcare Research and Quality. ${ }^{2,3}$ The overall strength evidence across studies was based on precepts outlined by the Grades of Recommendation Assessment, Development and Evaluation Working Group $^{4}$ and recommendations made by the Agency for Healthcare Research and Quality. 2,3

Details about methods can be found in the online supplementary material.

\section{Results}

From 258 citations, 11 citations were evaluated for full-text review. Three retrospective cohort studies (all using historical cohorts) (class of evidence [CoE] III) examining the comparative effectiveness and safety of LLIF/XLIF/DLIF versus PLIF/TLIF surgery and one prospective cohort study (CoE II) and two retrospective cohort studies (CoE III) reporting predictive factors following XLIF surgery met the inclusion criteria and form the basis for this report (-Fig. 1). Characteristics of studies investigating the comparative effectiveness of the surgical techniques are outlined in - Table 1 and those examining predictive factors following LLIF/XLIF/DLIF are outlined in -Table 2. Refer to the online supplementary material for critical appraisal, a list of excluded articles, and detailed outcome tables.

\section{Comparative Effectiveness of LLIF/XLIF/DLIF versus PLIF/TLIF}

None of the included studies reported radiographic or patient-reported outcomes for both treatment groups.

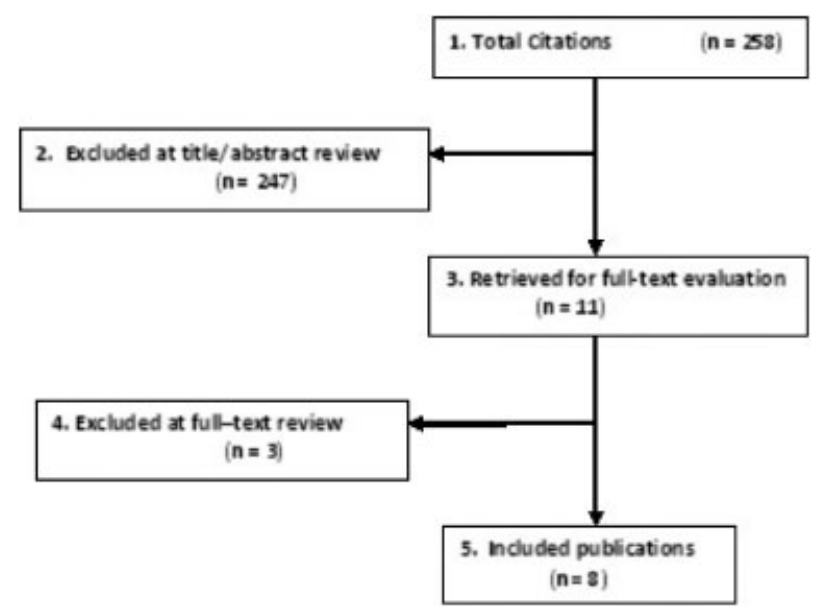

Fig. 1 Flow chart showing results of literature search. 


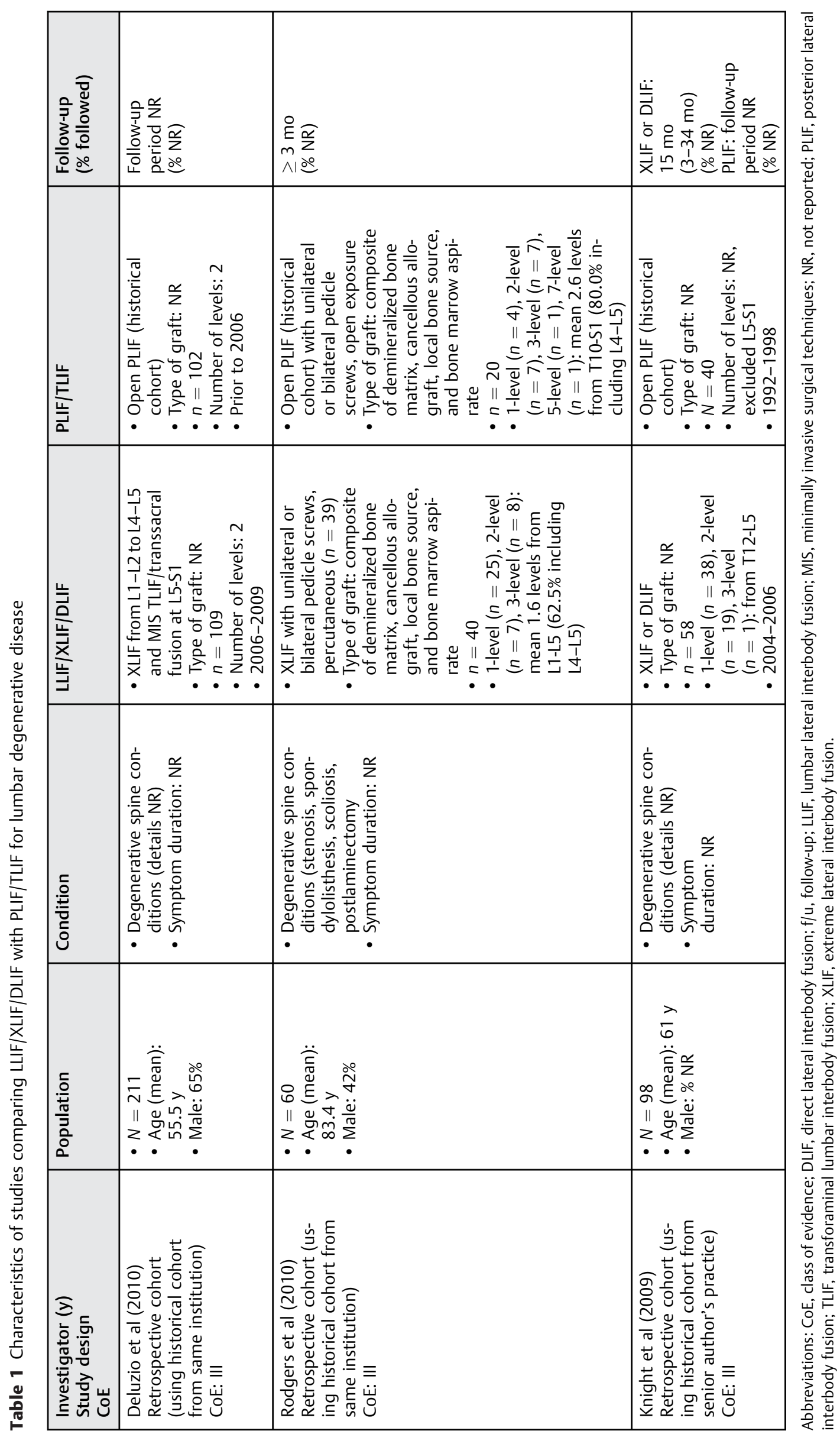




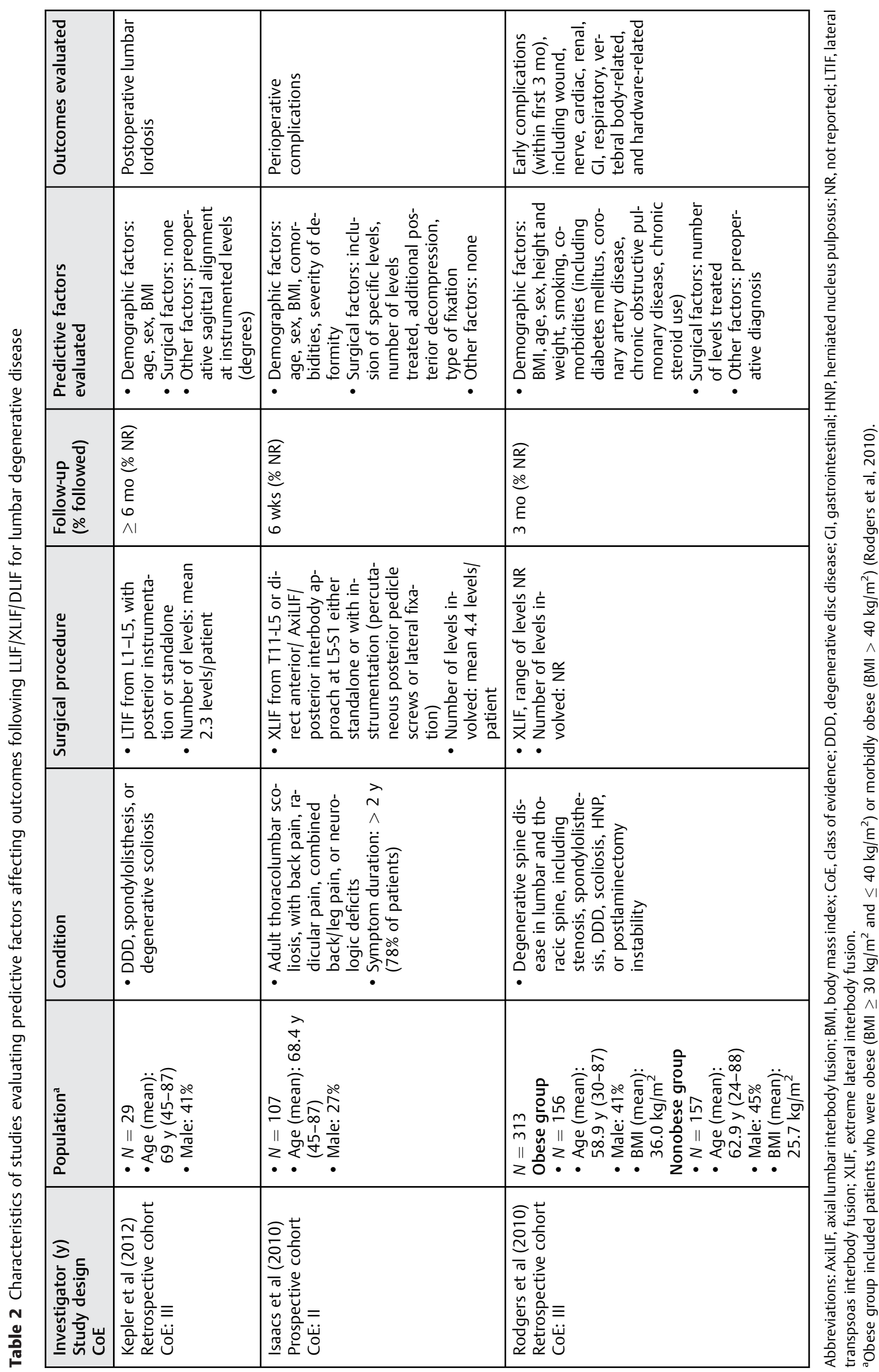


Table 3 Studies comparing LLIF/XLIF/DLIF with PLIF/TLIF: perioperative outcomes

\begin{tabular}{|c|c|c|c|}
\hline & LLIF/XLIF/DLIF & PLIF/TLIF & $p$ Value \\
\hline \multicolumn{4}{|l|}{ Length of hospital stay } \\
\hline Deluzio et al (2010) & $1.2 \mathrm{~d}$ & $3.2 \mathrm{~d}$ & NR \\
\hline Rodgers et al (2010) & $1.3 \mathrm{~d}$ & $5.3 \mathrm{~d}$ & $<0.0001$ \\
\hline Knight et al (2009) & $5 d(1-12)$ & $5 d$ & NS \\
\hline \multicolumn{4}{|l|}{ Estimated blood loss } \\
\hline Rodgers et al $(2010)^{a}$ & $1.4 \mathrm{~g}$ & $2.7 \mathrm{~g}$ & $<0.0001$ \\
\hline Knight et al (2009) & $136 \mathrm{~mL}$ & $489 \mathrm{~mL}$ & 0.0000 \\
\hline
\end{tabular}

Abbreviations: DLIF, direct lateral interbody fusion; LLIF, lumbar lateral interbody fusion; NR, not reported; NS, not significant; PLIF, posterior lateral interbody fusion; TLIF, transforaminal lumbar interbody fusion; XLIF, extreme lateral interbody fusion.

aBlood loss measured by average preoperative to postoperative hemoglobin change (Rodgers et al, 2010).

\section{Comparative Safety of LLIF/XLIF/DLIF versus PLIF/TLIF}

\section{Perioperative Outcomes}

- Length of hospital stay was reported by all three studies and found to be shorter in the LLIF group compared with the PLIF group in two studies ( - Table 1, - Supplementary Table 1, - Table 3, - Fig. 2). ${ }^{5,6}$

- Estimated blood loss measured by two different methods was reported by two studies and found to be significantly less in the LLIF group compared with the PLIF group in both studies. $^{6,7}$

\section{Reoperation Risks}

- Only one study reported reoperation risks for both treatment groups, with the LLIF group experiencing a lower reoperation risk compared with the PLIF group (-Table 4). ${ }^{6}$

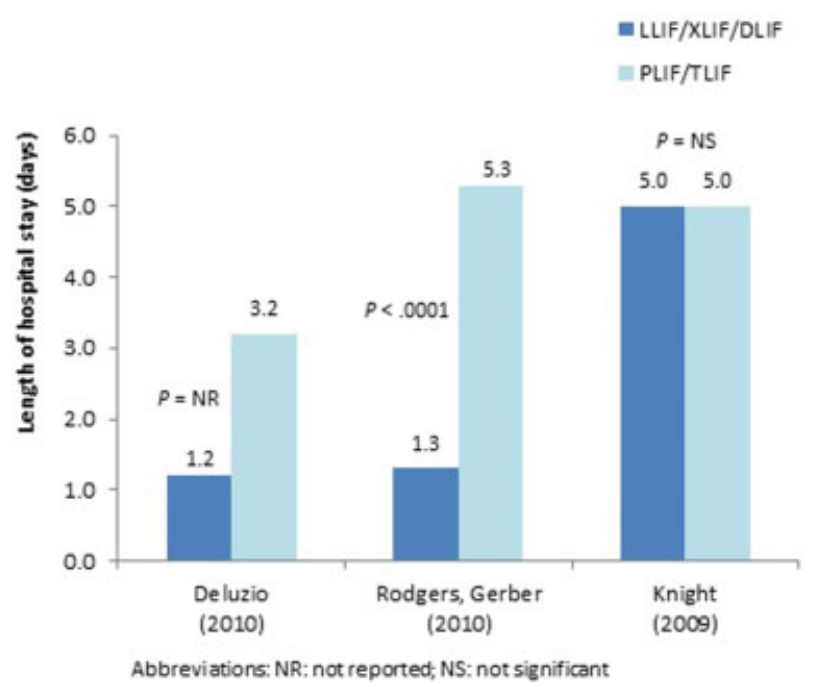

Fig. 2 Length of hospital stay for XLIF versus PLIF studies. NR, not reported; NS, not significant; DLIF, direct lateral interbody fusion; LLIF, lumbar lateral interbody fusion; PLIF, posterior lateral interbody fusion; TLIF, transforaminal lumbar interbody fusion; XLIF, extreme lateral interbody fusion.
- Only one study ${ }^{8}$ reported early reoperation for complications after XLIF; however, no specific reoperation timeframe is reported in the available studies.

- Five studies ${ }^{7-11}$ reported data on reoperations either following posterior open procedures ${ }^{9}$ or lumbar lateral interbody fusion. $7,8,10,11$

\section{Complications}

- Overall, complication risks ranged from 7.5 to $22.4 \%$ in the LLIF group and from 22.5 to $60.0 \%$ in the PLIF group in two studies (-Table 4, - Figs. 3 and $\mathbf{4}$ ). ${ }^{6,7}$

- Neurological complications following LLIF were reported in three studies, ${ }^{7-9}$ ranging from $0.9 \%$ of treated cases in one study ${ }^{7}$ to $13.8 \%$ in another study. ${ }^{9}$

- Mortality was higher in those with open PLIF (mean age, 84.2 years) compared with XLIF (mean age, 82.6 years) in one study (30 vs. $2.5 \%)^{6}$ but not different in another. ${ }^{7}$ The patient population experiencing the higher mortality risk were older compared with patients in the other study. In addition, the study with higher mortality risk was conducted at the same institution ${ }^{6}$ but not necessarily by the same surgical group as in the study with the lower mortality risk. ${ }^{7}$

Factors Affecting Patient Outcome after LLIF/XLIF/DLIF Surgery

Three factors were found to be associated with various poor outcomes following surgery (-Table 2, -Supplementary Table 2, - Table 5).

- Number of levels treated. There was 59\% increase in the complication risk for each additional level treated (odds ratio, $1.59 ; p=0.0105){ }^{9}$

- Preoperative diagnosis. Higher complication risks were reported in patients with a diagnosis of DDD or recurrent disc herniation (vs. scoliosis, spondylolisthesis, stenosis, or postlaminectomy instability) $(p=0.0075) .^{8}$

- Preoperative alignment. Preoperative alignment was significantly correlated with postoperative lordosis and increase in lordosis ( $p=0.003, p<0.001$, respectively). ${ }^{12}$ The levels with the least preoperative lordosis gained the most lordosis after surgery. 
Table 4 Studies comparing LLIF/XLIF/DLIF with PLIF/TLIF: reoperation risks and adverse events

\begin{tabular}{|c|c|c|c|}
\hline & LLIF/XLIF/DLIF, \% & PLIF/TLIF, \% & $p$ Value \\
\hline \multicolumn{4}{|c|}{ Reoperation risks and cause } \\
\hline Rodgers et al (2010) & $\begin{array}{l}5.0(2 / 40) \\
\text { compression fracture or NR }\end{array}$ & $\begin{array}{l}15.0(3 / 20) \\
\text { deep wound infection or } \\
\text { compression fracture }\end{array}$ & NS \\
\hline Knight et al (2009) & $\begin{array}{l}1.7(1 / 58) \\
\text { loss of fixation at } L 2-L 3 \text { for } \\
\text { acute subsidence }\end{array}$ & NR & \\
\hline \multicolumn{4}{|c|}{ Overall complication risk } \\
\hline Rodgers et al (2010) & $7.5(3 / 40)$ & $60.0(12 / 20)$ & $<0.0001$ \\
\hline Knight et al (2009) & $22.4(13 / 58)$ & $22.5(9 / 40)$ & NR \\
\hline \multicolumn{4}{|l|}{ Mortality risk } \\
\hline Rodgers et al (2010) & $2.5(1 / 40)$ & $30(6 / 20)$ & 0.0018 \\
\hline Knight et al (2009) & $0(0 / 58)$ & $2.5(1 / 40)$ & NR \\
\hline
\end{tabular}

Abbreviations: DLIF, direct lateral interbody fusion; LLIF, lumbar lateral interbody fusion; NR, not reported; NS, not significant; PLIF, posterior lateral interbody fusion; TLIF, transforaminal lumbar interbody fusion; XLIF, extreme lateral interbody fusion.

\section{Clinical Guidelines}

None found.

\section{Evidence Summary}

Overall, there is insufficient evidence of the comparative effectiveness of LLIF surgery versus PLIF surgery. There is low-quality evidence suggesting that LLIF surgery results in fewer complications or reoperations than PLIF surgery. And there is insufficient evidence that any factors exist that predict patient outcome after LLIF surgery (-Table 6).

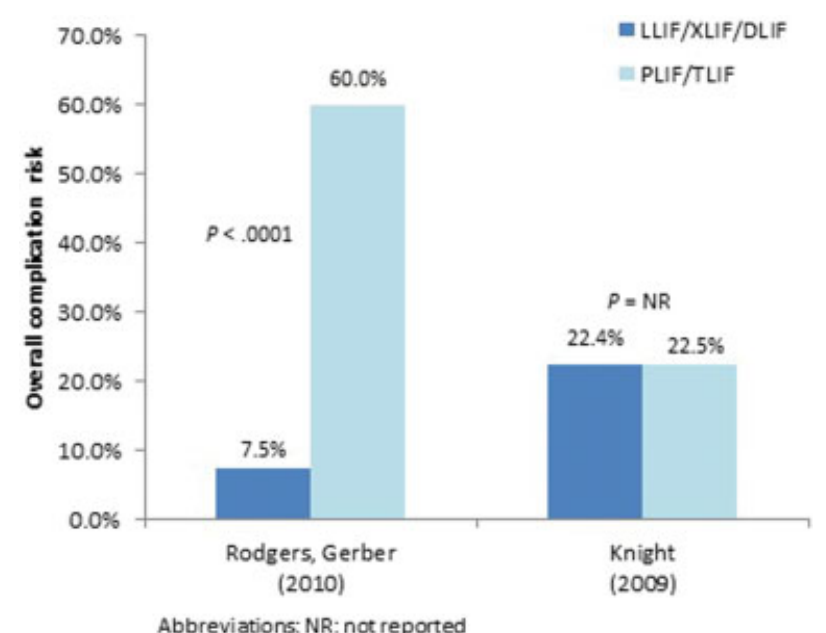

Fig. 3 Overall complication risks for XLIF versus PLIF studies. NR, not reported; DLIF, direct lateral interbody fusion; LLIF, lumbar lateral interbody fusion; PLIF, posterior lateral interbody fusion; TLIF, transforaminal lumbar interbody fusion; XLIF, extreme lateral interbody fusion.

\section{Illustrative Case}

A 65-year-old woman, with no significant medical history, presented with a long history of severe pain in the lumbar spine. No radicular pain was present. Symptoms made her ambulation difficult, as well as performing daily domestic activities.

Imaging revealed a degenerative thoracolumbar, leftsided convex scoliosis with apex at L2-L3 and L1-L2 (-Figs. 5 and 6).

She was initially treated with conservative care and medical drugs but without clinical benefit. Surgery was performed

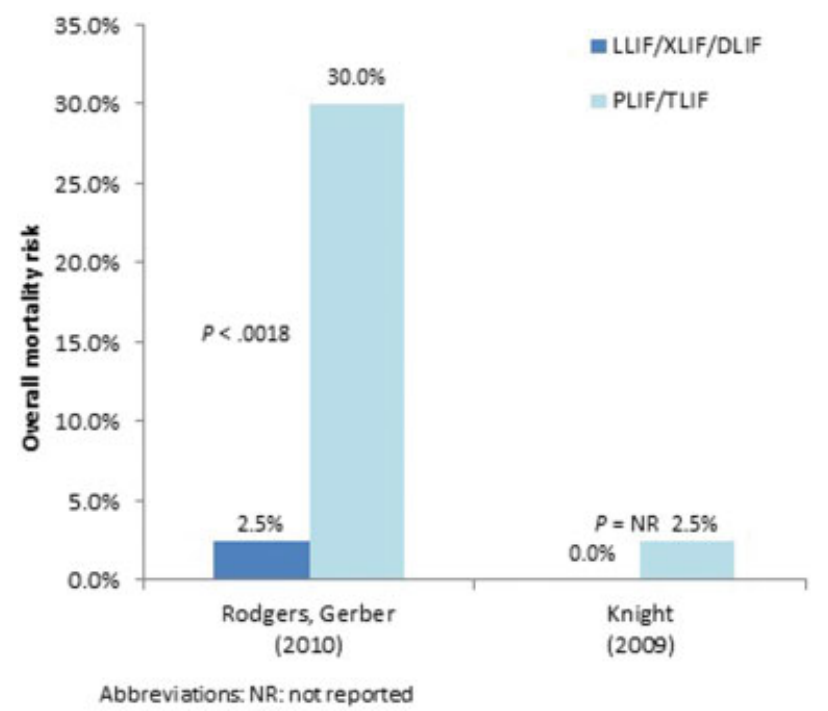

Fig. 4 Overall mortality risks for XLIF versus PLIF studies. NR, not reported; DLIF, direct lateral interbody fusion; LLIF, lumbar lateral interbody fusion; PLIF, posterior lateral interbody fusion; TLIF, transforaminal lumbar interbody fusion; XLIF, extreme lateral interbody fusion. 
34 Lumbar Lateral Interbody Fusion Barbagallo et al.

Table 5 Summary of demographic, surgical, and other factors evaluated as predictive factors for outcome following LLIF/XLIF/DLIF surgery

\begin{tabular}{|c|c|c|c|}
\hline & \multicolumn{2}{|c|}{ Multivariate analysis to control for confounders } & \multirow{2}{*}{$\begin{array}{l}\text { No multivariate analysis } \\
\text { Kepler et al (2012) }\end{array}$} \\
\hline & Isaacs et al (2010) & Rodgers et al (2010) & \\
\hline Outcome evaluated & Perioperative complications & Early complications & $\begin{array}{l}\text { Postoperative lumbar } \\
\text { lordosis }\end{array}$ \\
\hline \multicolumn{4}{|l|}{ Demographic factors } \\
\hline Age & NS & NS & NS \\
\hline Sex & NS & NS & NS \\
\hline BMI & NS & NS & NS \\
\hline Height/weight & & NS & \\
\hline Smoking & & NS & \\
\hline Comorbidities & NS & NS & \\
\hline Severity of deformity & NS & & \\
\hline \multicolumn{4}{|l|}{ Surgical factors } \\
\hline No. of levels treated & $\uparrow$ & NS & \\
\hline Inclusion of specific levels & NS & & \\
\hline Type of fixation & NS & & \\
\hline Additional posterior decompression & NS & & \\
\hline \multicolumn{4}{|l|}{ Other factors } \\
\hline Preoperative diagnosis & & $\uparrow$ & \\
\hline Preoperative sagittal alignment & & & $\uparrow$ \\
\hline
\end{tabular}

Abbreviations: BMI, body mass index; DLIF, direct lateral interbody fusion; LLIF, lumbar lateral interbody fusion; NS, not significant; XLIF, extreme lateral interbody fusion; $\uparrow$, increased risk of outcome.

Note: Empty cell indicates that factor was not evaluated.

Table 6 Evidence summary

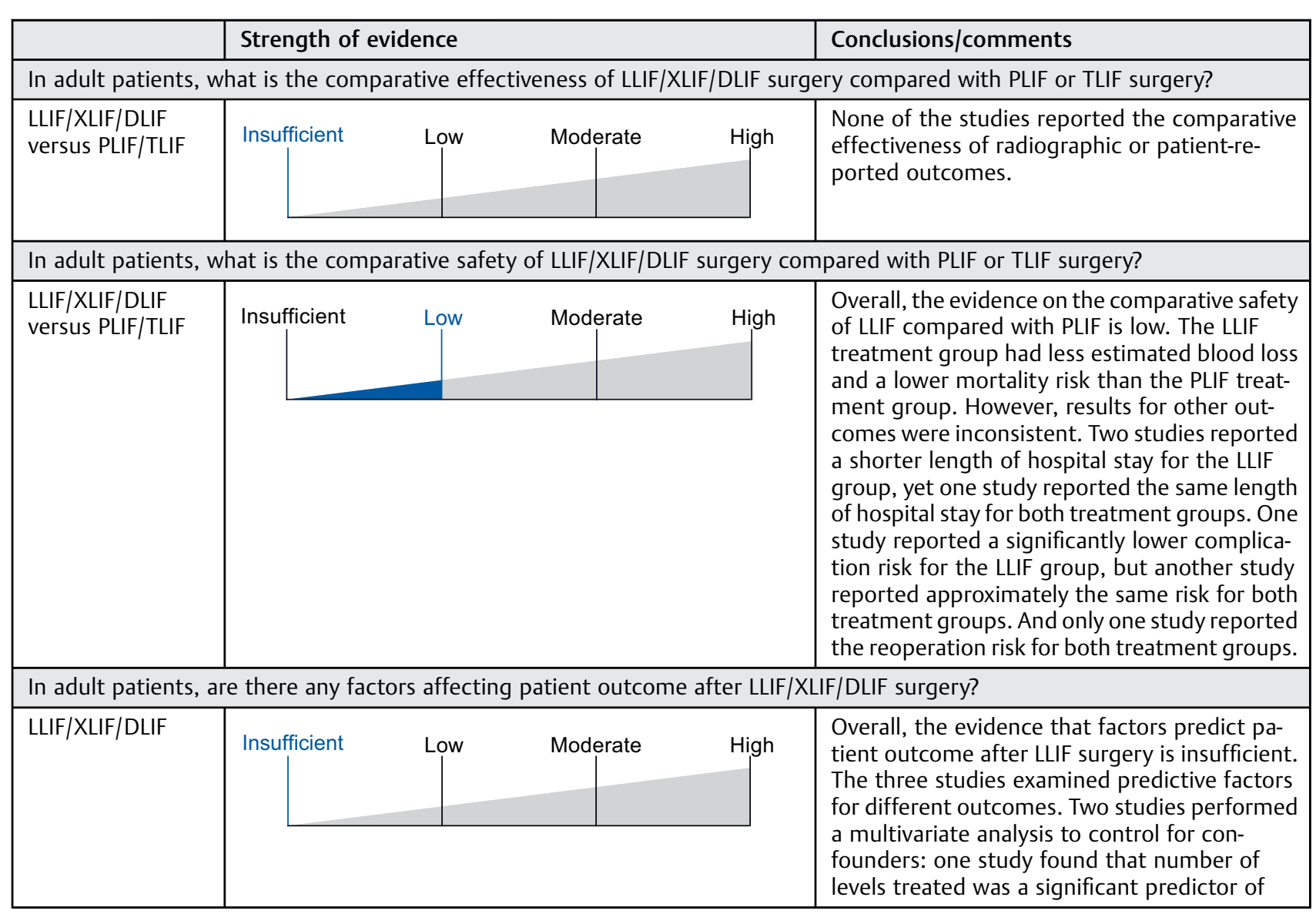


Table 6 (Continued)

\begin{tabular}{|l|l|l|}
\hline & Strength of evidence & Conclusions/comments \\
\hline & & $\begin{array}{l}\text { perioperative complications and one study } \\
\text { found that preoperative diagnosis was a sig- } \\
\text { nificant predictor of early complications. The } \\
\text { third study found that preoperative sagittal } \\
\text { alignment was a significant predictor of post- } \\
\text { operative lumbar lordosis but did not control } \\
\text { for confounders in the analysis. All three studies } \\
\text { found that age, sex, and BMI were not predic- } \\
\text { tors of outcome after LLIF. }\end{array}$ \\
\hline
\end{tabular}

Abbreviations: BMI, body mass index; DLIF, direct lateral interbody fusion; LLIF, lumbar lateral interbody fusion; PLIF, posterior lateral interbody fusion; TLIF, transforaminal lumbar interbody fusion; XLIF, extreme lateral interbody fusion.

Notes: Baseline strength: Risk of bias (including control of confounding) is accounted for in the individual article evaluations. High $=$ majority of articles level I/II; low = majority of articles level III/IV.

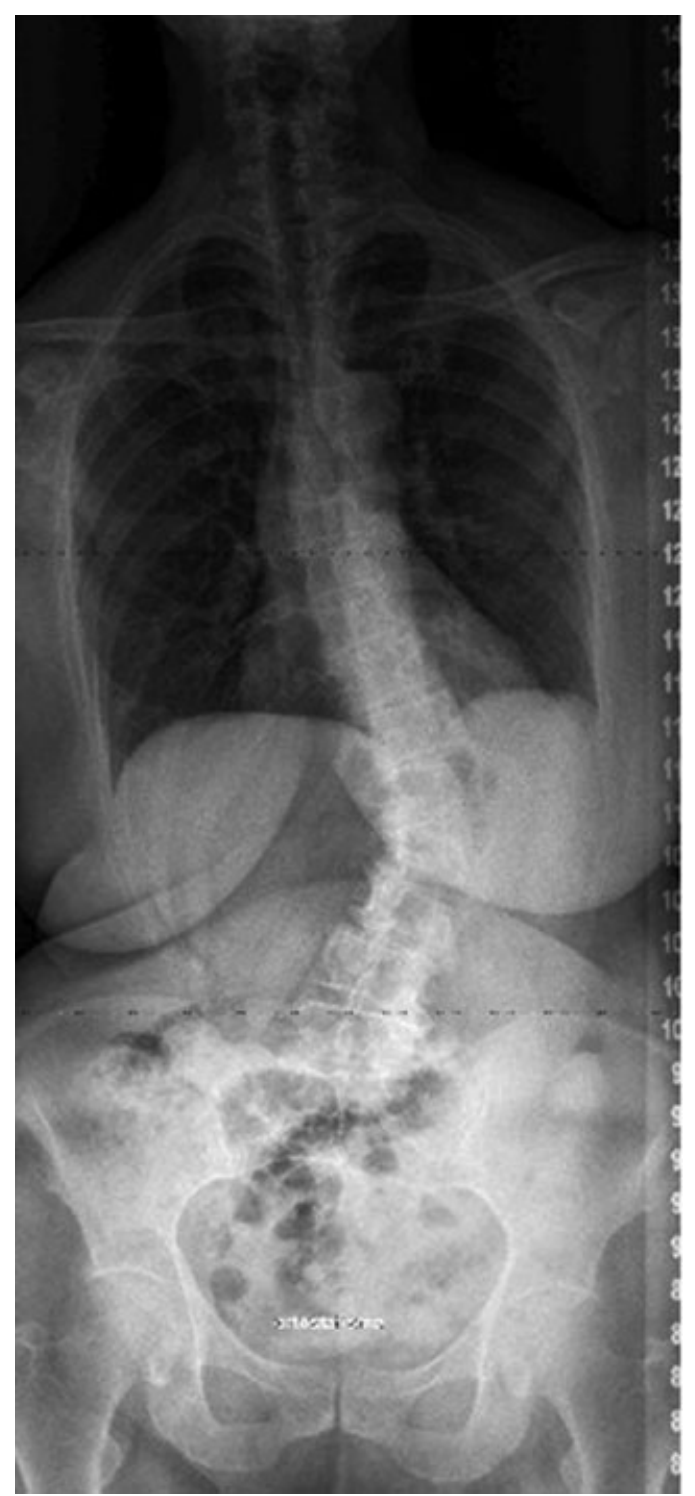

Fig. 5 Preoperative anterior posterior radiograph of 65-year-old female with degenerative lumbar scoliosis. with a two-level XLIF at the apex of the deformity (L2-L3, L1L2), followed by a posterior open correction and fixation from Th11 down to L5 (- Figs. 7 and 8).

Following such procedure, a good balance and alignment of the spine were obtained as well as improvement of pain. Full recovery of her domestic activity as well of ambulatory ability was achieved.

\section{Discussion}

- This systematic review is limited by the following:

- The majority of included studies were CoE III.

- There was a paucity of studies comparing LLIF surgery with PLIF or TLIF surgery.

- All three studies investigating the effectiveness of LLIF used historical controls who received PLIF or TLIF: two studies used a comparison group from the same institution ${ }^{5,6}$ and one study used a comparison group from the senior author's practice. ${ }^{7}$ Therefore, patients in the LLIF treatment group might have been subject to changes in policies or supportive care.

- The new direct lateral approach to the lumbar spine proves to be safe and effective, and at least comparable with the PLIF/TLIF techniques. This approach cannot be used for the L5/S1 level for anatomic limitations.

- The complications' rate shows to be inferior in the XLIF/ DLIF/LLIF compared with the PLIF/TLIF studies. ${ }^{6,7}$

- More studies with longer follow-up, including randomized trials, are necessary to evaluate the theoretical benefit of direct lumbar lateral approach and to assess whether the results of this strategy are superior and durable as the ones achieved by PLIF/TLIF technique performed in open or minimally invasive surgery.

- Potential limitations may also be related to some authors' conflicts of interest. $6,7,9,10$

\section{Disclosures}

Analytic support for this work was provided by Spectrum Research, Inc. with funding from AOSpine. 


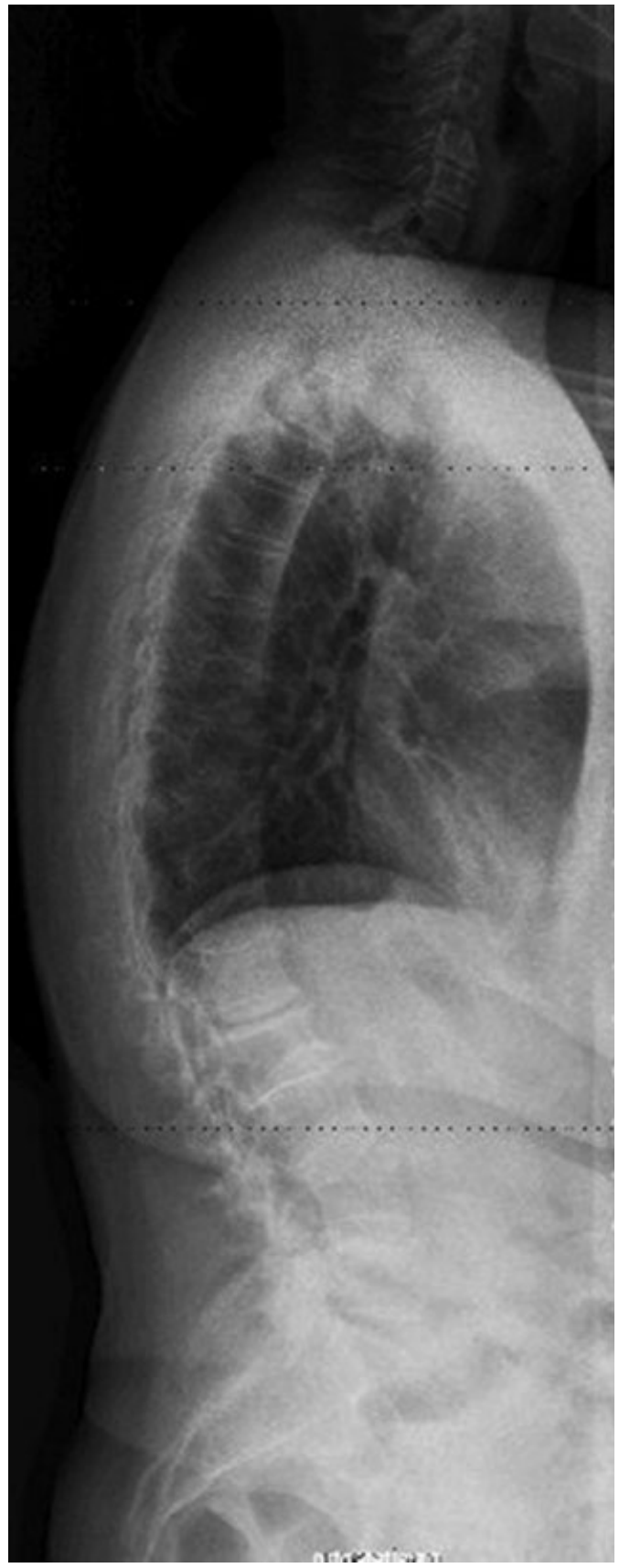

Fig. 6 Preoperative lateral radiograph.

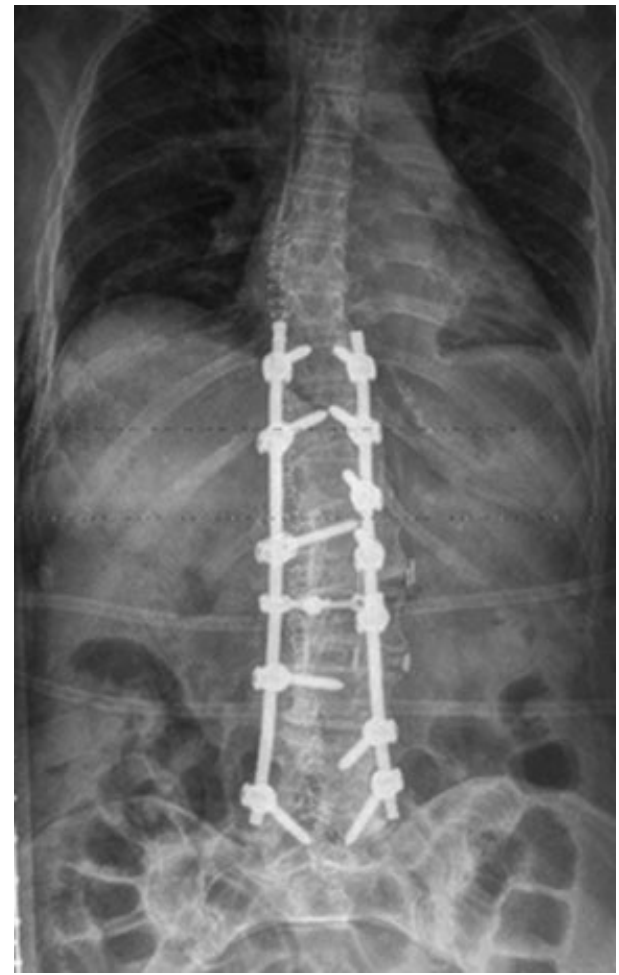

Fig. 7 Postoperative anterior posterior radiograph following two-level XLIF and posterior open correction and fixation.



Fig. 8 Postoperative lateral radiograph. 


\section{References}

1 Wright JG, Swiontkowski MF, Heckman JD. Introducing levels of evidence to the journal. J Bone Joint Surg Am 2003;85-A(1):1-3

2 Methods Guide for Effectiveness and Comparative Effectiveness Reviews AHRQ Publication No. 10(12)-EHC063-EF.2012; Available at: www.effectivehealthcare.ahrq.gov

3 West S, King V, Carey TS, et al. Systems to Rate the Strength of Scientific Evidence. Evidence Report/Technology Assessment No. 47 (Prepared by the Research Triangle Institute-University of North Carolina Evidence-based Practice Center, Contract No. 290-97-0011). Rockville, MD: Agency for Healthcare Research and Quality; 2002

4 Atkins D, Best D, Briss PA, et al; GRADE Working Group. Grading quality of evidence and strength of recommendations. BMJ 2004; 328(7454): 1490

5 Deluzio KJ, Lucio JC, Rodgers WB. Value and cost in less invasive spinal fusion surgery: lessons from a community hospital. SAS Journal 2010;4(2):37-40

6 Rodgers WB, Gerber EJ, Rodgers JA. Lumbar fusion in octogenarians: the promise of minimally invasive surgery. Spine 2010;35 (26, Suppl):S355-S360
7 Knight RQ, Schwaegler P, Hanscom D, Roh J. Direct lateral lumbar interbody fusion for degenerative conditions: early complication profile. J Spinal Disord Tech 2009;22(1):34-37

8 Rodgers WB, Cox CS, Gerber EJ. Early complications of extreme lateral interbody fusion in the obese. J Spinal Disord Tech 2010; 23(6):393-397

9 Isaacs RE, Hyde J, Goodrich JA, Rodgers WB, Phillips FM. A prospective, nonrandomized, multicenter evaluation of extreme lateral interbody fusion for the treatment of adult degenerative scoliosis: perioperative outcomes and complications. Spine 2010; 35(26, Suppl):S322-S330

10 Karikari IO, Nimjee SM, Hardin CA, et al. Extreme lateral interbody fusion approach for isolated thoracic and thoracolumbar spine diseases: initial clinical experience and early outcomes. J Spinal Disord Tech 2011;24(6):368-375

11 Caputo AM, Michael KW, Chapman TM, et al. Extreme lateral interbody fusion for the treatment of adult degenerative scoliosis. J Clin Neurosci 2013;20(11):1558-1563

12 Kepler CK, Huang RC, Sharma AK, et al. Factors influencing segmental lumbar lordosis after lateral transpsoas interbody fusion. Orthop Surg 2012;4(2):71-75

\section{Editorial Perspective}

The EBSJ reviewers felt that this topic was an excellent and timely choice made by the authors. There are many variants of the same idea being offered by industry, all of them based on the premise to avoid the extensor backside of patients while finding the magic interval between the lumbar plexus and the large vessels for the sake of decreased muscle dissection. Although the timing of this systematic review may appear premature to the point of offering the predictable "need more research" conclusion, this study does provide a valuable overview of the current state of research on this largely industry-driven technique-based procedure variant. That said, there is no doubt that the findings of this systematic review strongly support a comparative effectiveness-based project.

Criticisms of the reviewers revolved around the uncontrolled variables-curve deformity and subluxation, osteoporosis, previous surgery, and level of surgery. Access to the L4-5 level in particular can be difficult with lateral techniques due to the variable height of the pelvic crest and somewhat unpredictable bifurcation anatomy, leading to the question of how much "effectiveness" will mirror the rather positive "efficacy" results presented by experts in these early technique-based publications.

Finally, the reviewers raised the very critical point of potential for conflict of interest. As stated earlier, this technology is very clearly based on an industry "push." The question of the relationship of the investigators and the implant manufacturers, who are commonly newer to the market and tend to be more aggressive than the more established manufacturers, certainly warrants careful review of the disclosures made.

Finally, this is a very helpful status check and hopefully will help the EBSJ community advance their insights into this emerging surgical technique. 